\title{
Neutron imaging at PSI: a promising tool in materials science and technology
}

\author{
Eberhard H. Lehmann • Werner Wagner
}

Received: 25 March 2009 / Accepted: 24 February 2010 / Published online: 28 March 2010

(c) Springer-Verlag 2010

\begin{abstract}
Neutron imaging as a method for non-destructive studies takes advantage of the alternative contrast mechanism of neutrons compared to X-rays: most of the light elements feature high contrast, whereas heavy elements are relatively transparent to neutrons. In the previous decade, the neutron imaging technique has made substantial progress in well-tailored and well-equipped beam lines, in improvements of the image recording quality and efficiency by new and modern imaging techniques, and in modern image processing tools.

This article gives an overview of PSI's activities in this promising and still developing field combining high potential as research tool and as technique for industrial investigation and non-destructive testing. Examples are chosen from the fields of energy and mobility and inspection of highly activated samples. Furthermore, recent developments in phase contrast imaging and energy selective imaging studies are highlighted. Finally, future trends in neutron imaging are addressed.
\end{abstract}

\section{Introduction}

As directly visible in Fig. 1, neutron imaging and the traditional X-ray imaging deliver different, often complementary information from the same object. In this way, neutron imaging can be considered as an important complementary modern tool in non-destructive testing and investigations. Although the neutron imaging data look quite similar to Xray images it must be underlined that the interaction mecha-

E.H. Lehmann $(\bowtie) \cdot$ W. Wagner

Spallation Neutron Source Division, Paul Scherrer Institut, Villigen, Switzerland

e-mail: eberhard.lehmann@psi.ch nism with the sample material in neutron imaging is different from photon interaction: it is mostly caused by interaction with the atomic nuclei and not with the electrons as in the case of X-rays.

The application of neutron imaging studies to material research is manifold. For example, the non-destructive studies of welds, brazing, glue connections and corrosion are of industrial relevance, as well as the performance of electric fuel cells. The investigation of objects from cultural heritage like ancient sculptures opens new possibilities in different kinds of historical research. Furthermore, there are numerous applications in bioscience, geology, soil physics, nuclear power research and many others.

Although neutron radiography investigations are recognized as useful tools [1] since sufficiently strong neutron sources have been made available (about 60 years ago), the modern approach in neutron imaging differs dramatically from these early days experiments. There are at least three aspects which gave the push forward in this area: (i) the availability of advanced dedicated imaging beam lines at modern neutron sources, (ii) the development of digital neutron imaging systems and (iii) a profound understanding of the neutron transmission and scattering processes by simulations in combination with benchmarking experiments. The latter development was the basis for the quantification of imaging data and for neutron tomography in general.

State-of-the-art neutron imaging facilities should fulfill the following conditions:

- Dedicated beam line(s) at a strong neutron source

- High beam collimation $(L / D>500)$

- Well defined thermal or cold spectrum

- Low gamma background

- Large field of view (illumination field $\varnothing>20 \mathrm{~cm}$ )

- Space for experimental infrastructure inside a wellshielded area 

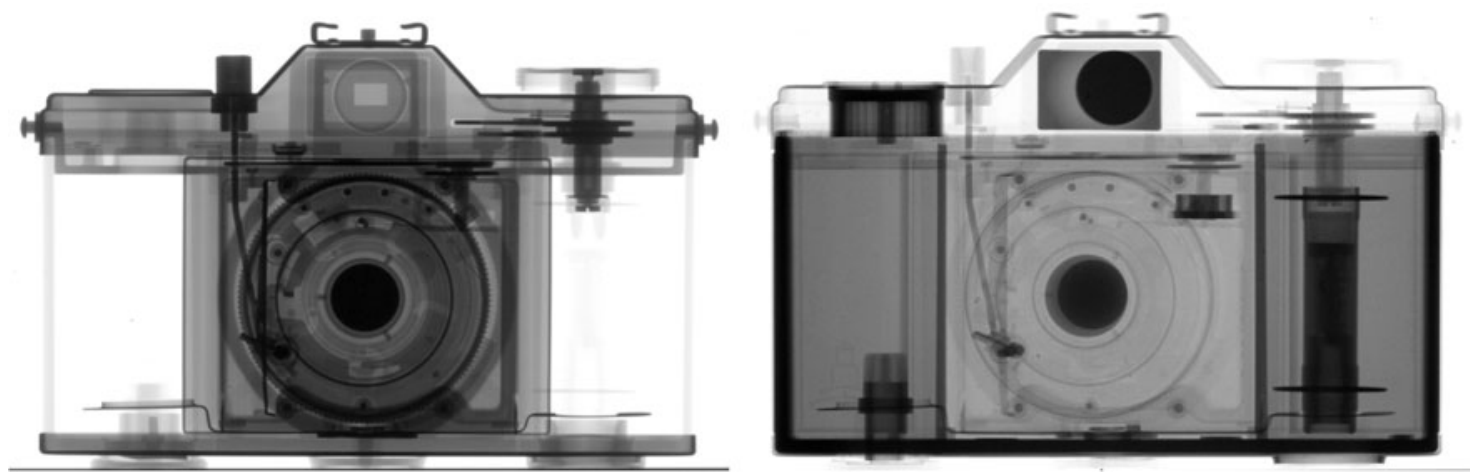

Fig. 1 The X-ray image (left) showing more the metallic parts and the neutron image (right) showing more the plastic parts (like the film reel) of a pocket camera. Both methods complement each other well in this case

- Digital imaging systems

- Remotely controlled sample manipulators

- User program, based on applied science and industrial partnership

The present overview describes the current situation in neutron imaging at the Swiss spallation neutron source SINQ (PSI, CH) with an outline about the facilities, the user program and some selected examples. It concludes with an outlook for further initiatives for methodical improvements and new challenging scientific applications.

\section{State-of-the-art capabilities of neutron imaging and tomography}

Among the 17 instruments installed at SINQ for different kinds of neutron applications there are two neutron imaging beam lines, one for thermal neutrons (NEUTRA [2]) the other for cold neutrons (ICON [3]). Figure 2 shows the layout of the ICON facility, which was set in operation in 2006.

As described in Table 1, the two facilities differ in their specific properties. Thus, one has the choice to decide from case to case which of the two instruments is most appropriate for a specific study. Both instruments are equipped with suitable digital detection systems, i.e. CCD cameras, imaging plates or a-Si flat panel detectors. At NEUTRA there is also an option for imaging with X-rays, called X-TRA [4], based on a $320 \mathrm{kV}$ X-ray tube, which can be swiveled into the beam line such that the imaging optics is the same as for the neutron beam. Thus, the X-ray data can be compared or combined pixel-wise with the neutron image data. X-TRA has the option as tomography device, as well. Due to the Xray beam geometry ( $3 \mathrm{~mm}$ focal spot, $8 \mathrm{~m}$ flight path) the parallel beam approximation is nearly perfectly fulfilled.

\section{Methods and performance}

NEUTRA and ICON were designed as flexible, general user instruments, serving a variety of scientific research and industrial applications, integrated in the PSI user program [5]. To satisfy the very different requirements it is necessary to be very flexible in respect to the beam conditions, detector options, inspection methods and the necessary infrastructure. With the help of variable apertures and different imaging positions along the beam line one can find an optimum balance between beam collimation (expressed by the L/D ratio), the beam intensity and the field of view for the specific experiments' requirements. All technical details can be found on the homepages of the facilities [6,7].

Recently [8], a micro-setup for tomography was made available for highest possible spatial resolution, close to the inherent limitations which are given by the intrinsic properties of the converter-scintillator detector, i.e. the range of secondary neutron capture products, which is of the order of some tens of microns. Under standard imaging conditions, the best achievable resolution is of that order, i.e. about $30 \mu \mathrm{m}$. In a special setup with a tilted detector an effective resolution of $10 \mu \mathrm{m}$ in one dimension was verified [9]. This has been proven to be very useful for electric fuel cell research to observe the electrochemical reaction in the membrane "edge-on" (see below).

There are some other features implemented, such as the option for the inspection of highly activated materials where a suitable shielding is required, scanning of large objects across the beam area by means of remotely controlled $x$ $y$-movable sample tables, and the infrastructure for gas supply (hydrogen, oxygen, nitrogen) and exhaust gas removal systems for the study of combustion related processes.

\section{Hot topic: energy end mobility}

Future mobility might not only be related to the combustion of gasoline or diesel fuel alone due to the limited resources 


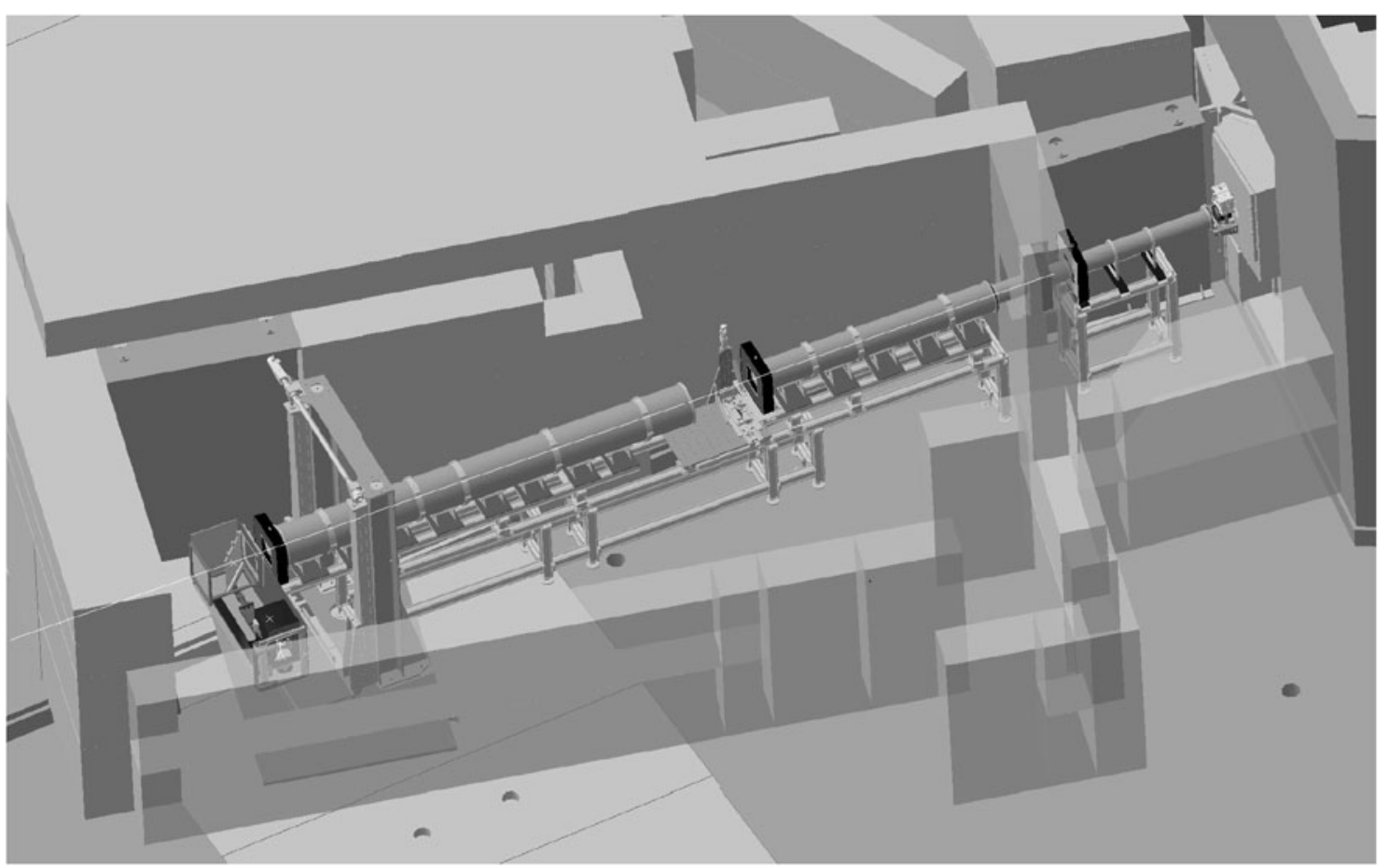

Fig. 2 Schematic view of the neutron imaging facility ICON [3]: neutrons from the cold source are delivered from the right where beam tailoring devices (apertures, shutter and filters) are hidden inside the shielding. Two positions can be used for dedicated investigations: the micro-tomography setup in the middle and the macro-position at the left end of the flight tube
Table 1 Some characteristic properties of the two neutron imaging beam lines at SINQ (the options in italic style are in preparation)

\begin{tabular}{ll}
\hline ICON & NEUTRA \\
\hline cold neutrons & thermal neutrons \\
higher contrast & higher penetration \\
variable aperture, Be-filter option & more homogenous illumination for large objects \\
two beam positions & two beam positions \\
three detector boxes & two detector boxes \\
energy selector & X-TRA option (320 kV tube, high current) for referencing \\
micro-Tomo-Setup & imaging plates infra-structure \\
differential phase contrast setup & tomography for macro-samples \\
energy selective option with single crystals & fuel cell infra-structure (hydrogen handling system)
\end{tabular}

and their environmental impact. New aspects are under consideration focusing on electric fuel cells, where hydrogen or methane and oxygen are combined in an electro-chemical reaction.

In this respect, one challenging research topic at PSI at present is the study the performance of electric fuel cells under various operational conditions. With the use of neutron radiography it is possible to investigate in real-time (up to $30 \mathrm{fps}=$ video standard) the water accumulation inside Polymer-Electrolyte Membrane Fuel Cells (PEMFC) with high spatial and temporal resolution. One example of such studies is illustrated in Fig. 3. Besides the high scientific interest in the electro-chemical processes there is important commercial relevance, since PEMFC are promising to re- place combustion based vehicle driving systems in the future on large scale.

Still, the majority of motorcars are based on combustion engines. Therefore the potential for optimization of the combustion process towards higher fuel efficiency and pollution reduction must be exploited intensively. Neutron imaging methods can certainly contribute to this task because the engine structure is relatively transparent for neutrons, allowing insight into the interior where the moving parts and the flow distribution of fuel- and/or lubricant can be imaged in realtime by stroboscopic triggering of the image recording. An example is illustrated in Fig. 4 (note: the motion of the inner elements can be followed in an animation, which is obtained as a stack of individual frames in a stroboscopic sequence). 
Fig. 3 Investigation of a model PEM fuel cell with neutron imaging: structural information is obtained in the left image, whereas the water distribution is extracted in a reference mode (right image). These measurements were done with the tilted detector option [9]. Visible is the membrane in the center $(50 \mu \mathrm{m})$ and the gas diffusion layer (GDL, $400 \mu \mathrm{m}$ ) around
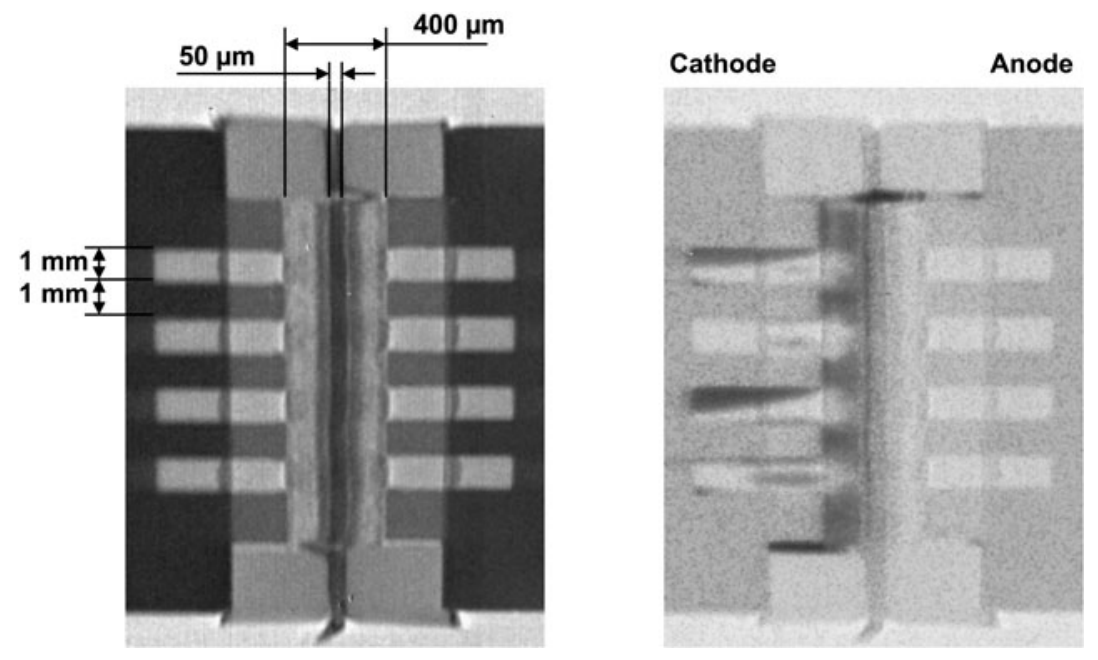

Fig. 4 Inspection of a cylinder head of a combustion engine with neutron tomography: the complex outer structure (left) can be visualized and measured and all inner components (right) as well. The most challenging task was to investigate the lubricant distribution inside the engine under operational conditions. This became possible due to the high contrast of hydrogenous materials (oil) in comparison to the relatively transparent metals (here $\mathrm{Al}$ ) in neutron imaging applications

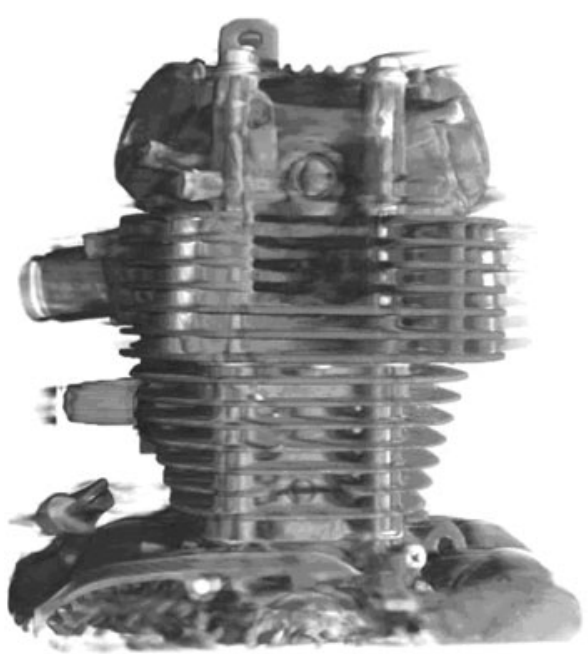

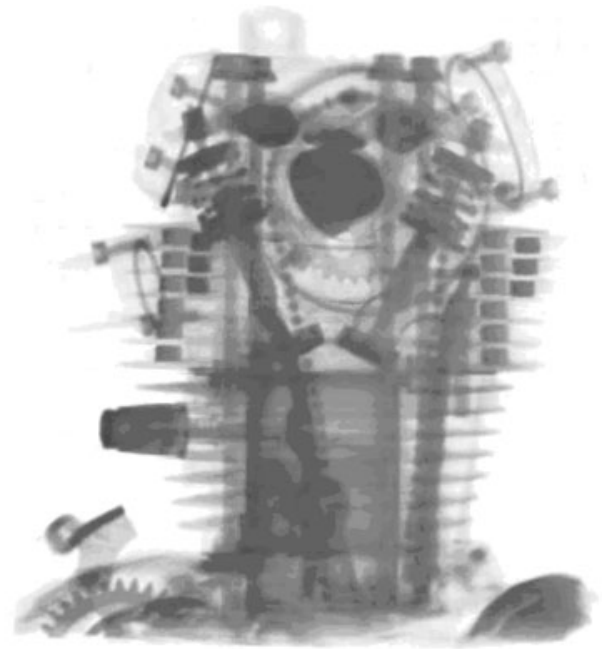

There are many aspects for further research using the presently available neutron imaging capabilities: lubricant distribution under stationary and real operational conditions, soot filter performance during loading and unloading phases, fuel injection during operation of engines, study of casting parts in respect to material homogeneity. Neutron imaging can further provide alternative information compared to other (more conventional) non-destructive techniques.

\section{Inspection of highly activated samples}

The neutron-producing target of the spallation source SINQ consists of an array of lead rods clad in steel, more recently in Zircaloy (see Fig. 5). Because of the necessity to investigate used targets with regard to their mechanical integrity and for lifetime assessment of future targets, neutron radiography of highly activated irradiated target rods became an issue of major importance.
For imaging these target rods, due to their high activity with dose rates on the Gy/h level, only an indirect imaging method can be used. Dy-loaded plates were used in the 1960's. When a neutron is captured, a beta-emitting radioactive isotope is created, which activates the plate that can be read by conventional X-ray sensitive photographic film. This method, although somewhat intricately, has successfully been used for nuclear fuel inspection, the burn up study of control rods or the study of pressure vessel material integrity [10].

A new approach for such kind of investigations is based on imaging plates containing Dy, embedded inside the neutron/gamma sensitive layer, as proposed and introduced by Tamaki [11, 12]. After exposure in the mixed neutron/gamma spectrum and subsequent erasure of the neutron/gamma-excitations, the image arising from the Dydecay can be read.

Such an image obtained from a lead rod containing a thermocouple at one side is shown in Fig. 6. Obviously, the thermocouple has been bent during operation, most likely by 


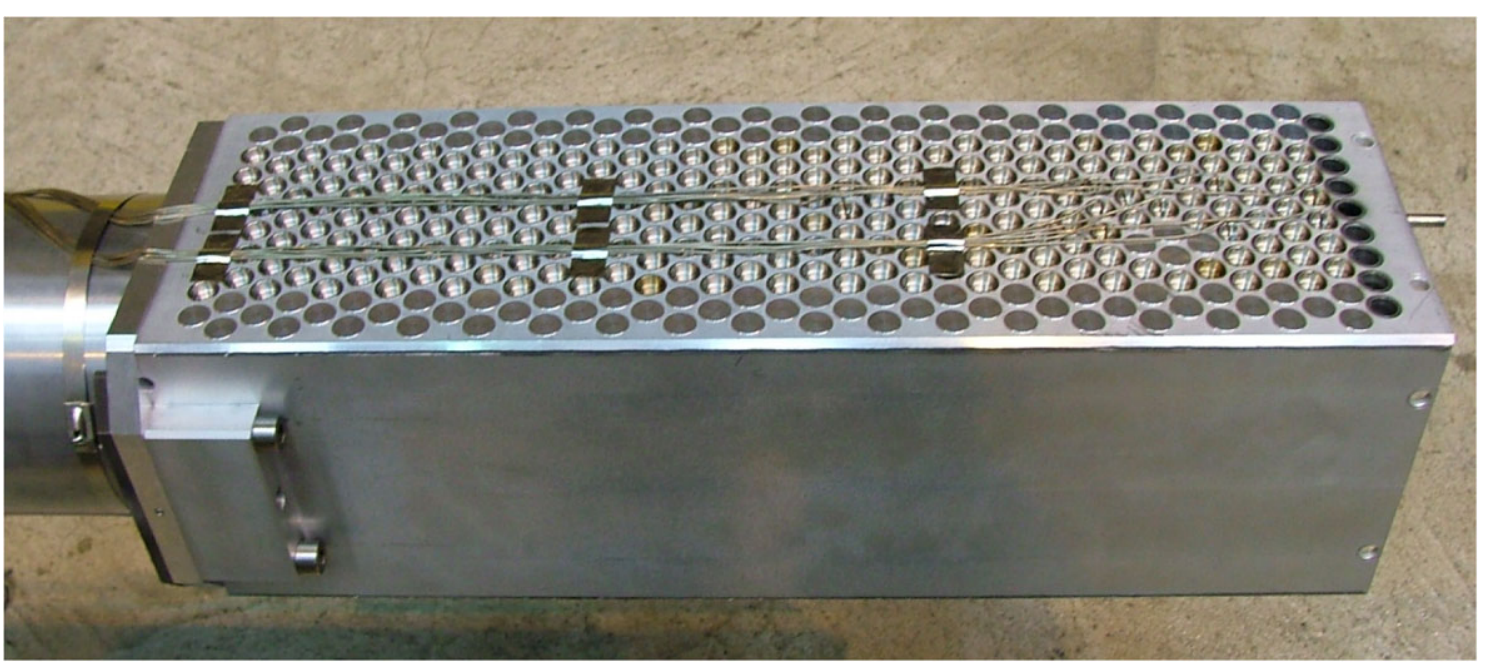

Fig. 5 The 5th spallation target of SINQ, consisting of $\mathrm{Zr}$ clad (outer rows) and steel clad (central rows) lead rods with space for water cooling in between. Some selected rods were inspected with neutron imaging methods before (not activated) and after operation (highly activated)

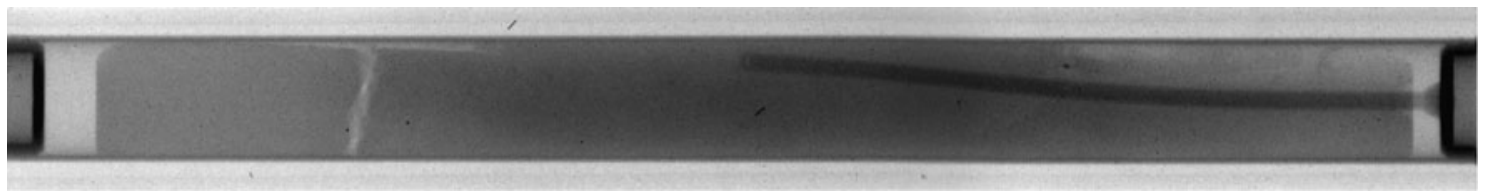

Fig. 6 Target rod of SINQ with lead filling after two years of proton exposure, inspected by neutron imaging methods using an adapted imaging plate method $[11,12]$. Watch the inserted thermocouple at the right and the crack in the lead filling at the left

levitation forces when the lead was partially melted. On the left side, a crack is visible in the lead filling, which occurred during solidification of the liquid lead. The darker area in the middle can be attributed to the accumulation of higher neutron absorbing spallation products, mostly $\mathrm{Hg}$. The obtained spatial resolution is relatively high $(0.05 \mathrm{~mm})$ and the contrast is directly related to the attenuation of the involved materials. This inspection showed no indication for corrosion or damage of the cladding hull, which would have limited the lifetime of such kind of target elements in the future. The amount of hydrogen in the cladding was found reasonably low and distributed homogenously.

\section{Phase contrast imaging}

Usually, neutron imaging investigations are performed in a sample-detector setup with only a small distance $d_{x}$ in between. In this way, the limitations with respect to spatial resolution caused by beam divergence can be kept small. The image blurring by geometrical reasons $u_{g}$ is described by the relation:

$u_{g}=\frac{d_{x}}{L / D}$.

If the beam is highly collimated $(L / D>1000)$, larger sample-detector distance can be tolerated. Furthermore, in

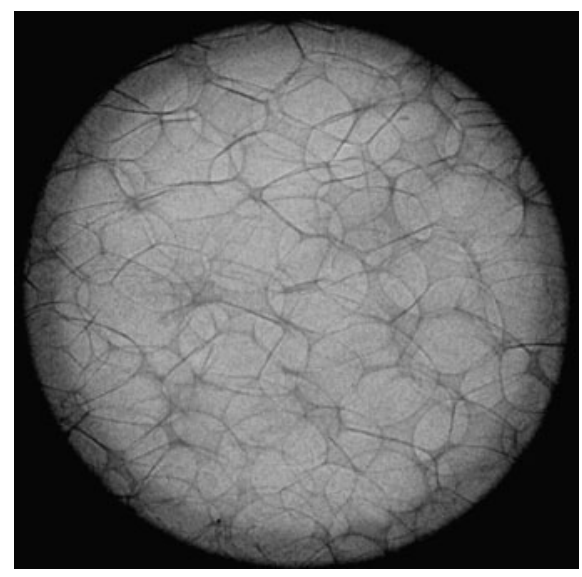

Fig. 7 Phase contrast image of $\mathrm{Al}$ foam: image diameter $4.5 \mathrm{~cm}$, exposure time $180 \mathrm{~min}$ on imaging plates, sample-detector distance $80 \mathrm{~cm}$; the experiment was performed at NEUTRA

such configuration interference features become visible in the neutron images as shown in Fig. 7: An edge enhancement occurs when the source point is small (typically $1 \mathrm{~mm}$ ) and far from the detector (about $10 \mathrm{~m}$ ), and the distance $d_{x}$ is selected appropriately. Such kind of imaging is called propagation based phase contrast. It can be particularly useful for weakly absorbing materials by enhancing the edge contrast at the interface between otherwise almost indistinguishable 
Fig. 8 Energy selective studies with a turbine selector device $(\Delta \lambda / \lambda \sim 15 \%)$ in the region near the Bragg edges of iron (bcc): a high contrast variation is obtained at about 4 Angstrom. The finite energy resolution is smoothing the edge
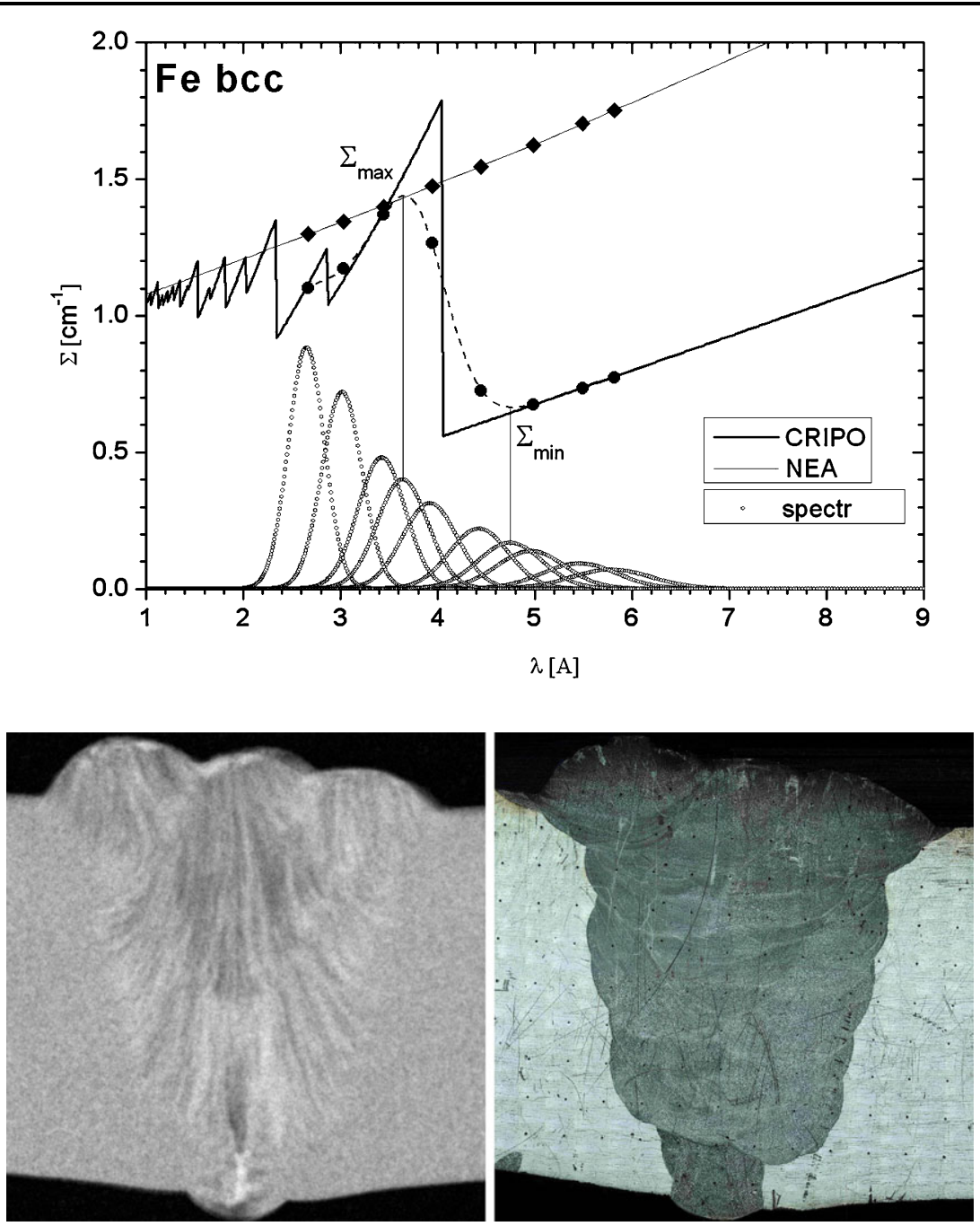

Fig. 9 Study of a steel weld in energy selective imaging mode at 4 Angstrom in transmission: special features become visible (left image) which are attributed to strain streaks resulting from the crystallization process during solidification. This bulk-structural information cannot be obtained from a surface analysis with etched polished surfaces (right: photo). The shown sample is $24 \mathrm{~mm}$ high features. An overview on the state-of-the-art of this method is given in [13].

There is another approach involving phase effects in imaging, i.e. the direct determination of the phase contribution to neutron-sample interaction. Considering the neutron propagation as wave type property, a complex neutron refraction index $n$ can be defined as:

$n=1-\delta+i \beta$.

The absorption based contribution $\beta$ can be determined in the "standard" attenuation setup, whereas the phase signal observation $(\delta)$ needs some special installation. As proposed and realized in [14], a separate phase signal for each sample material can be derived under certain conditions (in-line coherence, limited spectral range, phase stepping scanning with a grating interferometer). This method can be used to distinguish materials with about the same attenuation but different phase shift properties. An installation dedicated for phase contrast imaging is under way to be realized at the ICON beam line in due course.

\section{Energy selective neutron imaging}

The availability of cold neutrons at ICON opened another interesting method for material research, i.e. the study of structural materials ( $\mathrm{Fe}, \mathrm{Ni}, \mathrm{Ti}, \mathrm{Zr}, \mathrm{Al}, \mathrm{Cu}, \ldots$ ) in transmission mode near their Bragg edges. As shown in Fig. 8, the Bragg edges are most pronounced at wavelength around 4 Angstrom and above, depending on the individual material. This behavior has several consequences: the transmission and/or contrast vary by a factor 2 and more when the energy passes the Bragg edge of a specific element. On the other hand, scattering artifacts occur at several energies as illustrated by the example of a weld in Fig. 9, which gives indications about textured structure or strain streaks within the weld. These features result from the bulk and do not necessarily correspond to the structural information obtained at the weld surfaces after polishing and etching.

The energy resolution of a mechanical, turbine type velocity selector [15] is of the order of 10-15\%. As also shown in Fig. 8 this results in a smoother contrast variation at the 
Fig. 10 Head of a diesel injection nozzle, investigated with cold neutron tomography in high resolution mode: the outlet channels, only $0.15 \mathrm{~mm}$ in opening diameter, are well visible. Residual non-evaporated diesel fuel is imaged in high contrast (bright in the left picture)
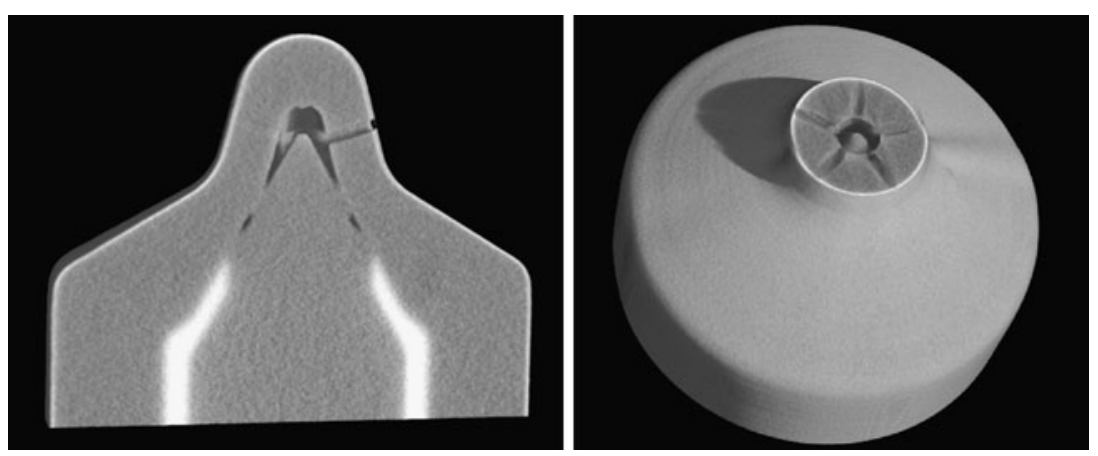

Bragg edges. Narrower energy resolution is obtained by using single crystals, however, on the expense of intensity. The most favorable approach for best energy/intensity selection can clearly be expected by the time-of-flight (TOF) technique at pulsed spallation neutron sources [16].

\section{Future trends in neutron imaging}

It is a real challenge to improve the spatial resolution in digital neutron imaging. The new high resolution tomography setup allows for new kinds of investigation also for industrial applications. As an example, the three-dimensional structural analysis of a diesel injection nozzle is shown in Fig. 10. It shows how well the duct channels in the steel head can be visualized and measured dimensionally. On the other hand, the remaining diesel fuel can be imaged with high contrast due to the high contrast of hydrogenous materials for neutrons.

With the improvement of the energy resolution in neutron imaging it might be possible to see also internal stresses in structural materials. The advantage to use direct imaging for this purpose is the much higher spatial resolution compared to the point-wise scanning with diffractive devices. Although the neutron intensity becomes smaller and smaller with narrowing energy bands this approach is still justified when the performance and the output is compared to the existing strain scanning devices. In the future, using the TOF option at the new, most powerful pulsed spallation sources, i.e. SNS in the US or JSNS in Japan, even tomography studies might be possible.

It was demonstrated first at ICON [14] that a device for differential phase contrast measurements based on a grating interferometer is promising to extract additional material information complementing the common absorption contrast. In the meantime more studies were done, i.e. for the detection of magnetic domains in bulk materials. A user device is under construction for ICON which will enable routine experiments for phase dependent material effects.

Imaging with polarized neutrons, as well, is a promising approach for the investigation of magnetic structures and phenomena. After first feasibility studies there is potential for improvement of the setup regarding spatial resolution, field of view and sensitivity for magnetic fields. The beam line FUNSPIN at SINQ [17], providing a polarized beam, can be modified in the future to a test device for polarized neutron imaging.

\section{Summary}

Neutron imaging is established at PSI as a well accepted research method within the suite of experimental devices around SINQ. The two well-equipped neutron imaging facilities deliver high flexibility in the user program and in the service for industrial partners. Some new and challenging approaches are in preparation or envisaged for the future progress of neutron imaging at SINQ: differential phase contrast, energy selection imaging, polarized neutron application, which after the successful installation will be made available to the user community.

\section{References}

1. Proc. World Conferences on Neutron Radiography 1-8, 19862006

2. E. Lehmann, H. Pleinert, The new neutron radiography station at the spallation source SINQ, INSIGHT 40(3) (1998)

3. G. Kühne, G. Frei, E. Lehmann, P. Vontobel, A. Bollhalder, U. Filges, M. Schild, ICON-the new facility for cold neutron imaging at the Swiss spallation neutron source SINQ, Swiss. Neutron News 28, 20-29 (2005). http://sgn.web.psi.ch/sgn/snn/snn_ 28.pdf

4. E. Lehmann, P. Vontobel, G. Frei, The approach of X-ray enhanced neutron imaging and its realization at the NEUTRA facility, PSI-Annual Report 2004, vol. III, p. 218

5. https://duo.psi.ch/duo/

6. http://neutra.web.psi.ch

7. http://neutra.web.psi.ch/facility/icon_index.html

8. E.H. Lehmann, G. Frei, G. Kühne, The micro-setup for neutron imaging: A major step forward to improve the spatial resolution. Nucl. Instrum. Methods Phys. Res. A 576(23), 389-396 (2007)

9. P. Boillat, G. Frei, E.H. Lehmann, G. Scherer, A. Wokaun, Neutron imaging resolution improvement optimized for fuel cell applications. Appl. Phys. Lett. (2009, submitted) 
10. J. Bakker, A. Baritello, J. Bordo, J. Markgraf, H. Leeflang, I.I. Ruyter, Neutron radiography of light water feuel rods at the high flux reactor Petten, in Proc. 2nd World Conference on Neutron Radiography, Paris, 1986 (Reidel, Dordrecht, 1986)

11. M. Tamaki, K. Iida, N. Mori, E. Lehmann, P. Vontobel, M. Estermann, Nucl. Instrum. Methods Phys. Res. A 542, 320-323 (2005)

12. P. Vontobel, M. Tamaki, N. Mori, T. Ashida, L. Zanini, E. Lehmann, M. Jaggi, Post-irradiation analysis of SINQ target rods by thermal neutron radiography. J. Nucl. Mater. 356(13), 162-167 (2006)

13. K. Lorenz, Implementation of neutron phase contrast imaging at FRM-II, Dissertation, TU Munich, 2008
14. F. Pfeiffer, C. Grünzweig, O. Bunk, G. Frei, E. Lehmann, C. David, Neutron phase imaging and tomography. Phys. Rev. Lett. 96, 215505 (2006)

15. V. Wagner, H. Friedrich, P. Wille, Physica B 180-181, 938 (1992)

16. W. Kockelmann, G. Frei, E.H. Lehmann, P. Vontobel, J.P. Santisteban, Energy-selective neutron transmission imaging at a pulse source. Nucl. Instrum. Methods Phys. Res. A 578, 421-434 (2007)

17. J. Zeima, G. Ban, M. Beck, A. Bialek, G. Frei, C. Hilbes, G. Kühne, P. Gorel, K. Kirch, S. Kistryn, M. Kuzniak, A. Lindroth, O. Naviliat-Cuncic, J. Pulut, N. Serverijns, E. Stephan, FUNSPIN polarized cold neutron beam at PSI. Nucl. Instrum. Methods A 539, 622-639 (2005) 